\title{
Análisis del papel del turismo residencial en la cabecera municipal de Ixtapan de la Sal, Estado de México
}

\author{
Lic. en T. Esteban Arias
}

\begin{abstract}
RESUMEN
El presente artículo es resultado de un trabajo de tesis para la obtención del título de licenciatura. El propósito del estudio fue analizar los efectos que genera el turismo residencial en Ixtapan de la Sal a fin de conocer de manera puntual la incidencia en el desarrollo turístico de este destino. El método aplicado en este trabajo, fue el clínico y se empleó con la finalidad de conocer la situación actual del turismo residencial. Así también, con el método deductivo emanado de la revisión teórica de la propuesta de Daniel Hiernaux en su obra Segundas Residencias fue posible lograr confrontar su teoría con los datos recogidos en campo. A partir de la década de los cuarenta, el turismo en México ha presentado distintas modalidades: el turismo de sol y playa; el turismo religioso o el ecoturismo; particularmente, el turismo residencial es una tendencia de reciente auge, práctica aún cuestionada como modalidad turística por no hacer uso de los servicios de alojamiento hotelero como lo hace el turismo convencional, pero que tiene repercusiones importantes en las comunidades anfitrionas, como es el caso de estudio.
\end{abstract}

PALABRAS CLAVE

Turismo residencial, desarrollo turístico, sistema turístico. 


\title{
Analysis on the role of residential tourism in the municipality of Ixtapan de la Sal in the Estado de Mexico
}

\author{
Lic. en T. Esteban Arias
}

RESUMEN

This article is the result of a thesis project to obtain the bachelor's grade. The purpose of study was to analyse the effects that are generated by residential tourism in Ixtapan de la Sal with the intension of knowing accurately the significance on the tourisms development of this destination. The method used in this project was the clinic approach. It intended to know the present situation of residential tourism. In addition, with the deductive method emerged from the theoretical revision proposed by Daniel Hiernaux in his work Segundas Residencias it was possible to confront theory and results extracted from field research. From the 40s on, tourism in Mexico has shown different modalities: sun and beach tourism, religious tourism, ecotourism and, particularly, residential tourism. A tendency of recent apogee, it is an activity which has been questioned as a tourism modality for it does not uses the hotel accommodation services as conventional tourism might do. However, it has important resonance on the host communities, as it is the case of study.

PALABRAS CLAVE

Residential tourism, tourism development, tourism system 


\section{INTRODUCCIÓN}

En México, el periodo que comprende de la década de los años cuarentas a los ochentas, el desarrollo turístico se concentró en el turismo de sol y playa como modalidad dominante. Sin embargo, a partir de la globalización han surgido nuevas tendencias, por ejemplo; el ecoturismo y el turismo religioso. El turismo residencial es una tendencia de reciente auge en México, aún cuestionada como modalidad turística por no hacer uso de los servicios de alojamiento hotelero como el turismo convencional.

El turismo residencial ha tenido distintas expresiones en nuestro país desde los primeros años de la gestación del turismo en la década de los años cuarentas, como es el caso del Lago de Chapala y Acapulco, entre otros. El Estado de México presenta una manifestación de este tipo de turismo desde sus primeras exposiciones que es importante analizar como fenómeno explicativo de una corriente de viajeros que pernocta en sus diferentes destinos turísticos. Para efectos de este trabajo se estudia el caso de Ixtapan de la Sal, por ser uno de los sitios turísticos de mayor consolidación en el estado y en el que se observa un reciente desarrollo del turismo residencial. 


\section{METODOLOGÍA}

Para fines de la investigación fue necesario el usó del método clínico, el cual persigue emitir un diagnóstico. Se aplicó con la finalidad de conocer la situación actual del turismo residencial en Ixtapan de la Sal. Además, la aplicación tanto de técnicas cuantitativas como cualitativas para el trabajo de campo, entre las cuales mencionaremos las siguientes:

- En una primera fase se llevó a cabo un trabajo de recorrido e identificación visual de áreas ocupadas por el turismo residencial y sus actividades afines. Posteriormente, se aplicaron entrevistas a las autoridades municipales para obtener mayor información sobre las segundas residencias.

previo conocimiento de las zonas residenciales.

- Diseño y aplicación de un cuestionario específico para personal que trabaja en segundas residencias.

- Diseño y aplicación de cuestionarios para prestadores de servicios turísticos, así como de comercios y servicios diversos, para identificar su relación con el turista residencial.

- Diseño y aplicación de cuestionarios para comercios y servicios sobre diversos giros económicos.

- Diseño y aplicación de cuestionarios para turistas residenciales.

La aplicación de estos cuestionarios se llevó a cabo en base a la disponibilidad misma de las personas; es decir, dependiendo del tiempo y actitud de los encuestados, ya que su tiempo era limitado por motivos de trabajo (comerciantes y prestadores de servicios), la falta de permiso de los dueños (empleados de las segundas residencias) o estadía por motivos de descanso (turistas residenciales). Los datos obtenidos con los cuestionarios sirvieron para conocer el perfil del turista residencial y su relación con comercios diversos y prestadores de servicios turísticos. 


\section{TURISMO RESIDENCIAL}

(acercamiento conceptual)

Este estudio se adentra en una de las modalidades de turismo menos estudiadas en México, pero con gran participación en toda la República Mexicana, sin importar que el destino tenga una total vocación turística.

En México, el periodo que comprende de la década de los años cuarenta a los ochenta, el desarrollo turístico se concentró en el turismo de sol y playa como modalidad dominante. Sin embargo, a partir de la globalización han surgido nuevas tendencias, por ejemplo, el ecoturismo y el turismo de aventura. El turismo residencial es una tendencia de reciente auge en México, aún cuestionada como modalidad turística por no hacer uso de los servicios de alojamiento hotelero como el turismo convencional.

El turismo residencial ha tenido distintas expresiones en nuestro país desde los primeros años de la gestación del turismo en la década de los años cuarenta, como es el caso del Lago de Chapala y Acapulco, entre otros. El Estado de México presenta una manifestación de este tipo de turismo desde sus primeras exposiciones que es importante analizar como fenómeno explicativo de una corriente de viajeros que pernocta en sus diferentes destinos turísticos. Para efectos de este trabajo se estudia el caso de Ixtapan de la Sal, por ser uno de los sitios turísticos de mayor consolidación en el estado y en el que se observa un reciente desarrollo del turismo residencial.

Según la Organización Mundial de Turismo (OMT), “El turismo es el conjunto de relaciones y fenómenos productivos por el desplazamiento y permanencia de personas fuera de su lugar de domicilio, en tanto dichos desplazamientos y permanencia no estén motivados por una actividad lucrativa" (OMT;1995:10). Ésta definición muestra la esencia misma del fenómeno en cuestión, ya que marca puntos importantes, por ejemplo, el desplazamiento que se tiene que realizar hacia otro fuera del lugar de origen o residencia, con la finalidad de practicar alguna actividad no rentable durante la estancia en el destino, 
además de hacer uso de los servicios que éste proporcione, como alojamiento, alimentación y entretenimiento.

Para Montaner (1998:371), el turismo de segundas residencias es aquel que "se caracteriza por su estabilidad en el lugar de destino. aunque no exclusivamente, el lugar de residencia escogido es frecuentemente un hotel, apartamento, segunda residencia, villa, chalet, o similar".

Según Herrera Romero (1997:43), el turismo residencial "es la modalidad particular del turismo que se caracteriza por un pronunciado conjunto de relaciones y fenómenos que se suscitan por el desplazamiento voluntario y periódico, de su lugar de residencia habitual hacia una residencia secundaria por un motivo de descanso y recreación".

El turismo residencial es una forma especial de hacer turismo, ya que tiene características particulares, dentro de las cuales se tiene que el turista no hace uso de servicios de alojamiento tradicional, el poder adquisitivo de los turistas es alto, en virtud de que se debe cubrir el mantenimiento de una segunda residencia, la principal actividad que realiza el turista es el descanso, y por lo general la estancia es realizada los fines de semana o puentes, dichas cualidades lo distinguen del resto de la actividad turística.

A diferencia de la definición tradicional de turismo, se refuerzan dos puntos centrales: el primero y el más importante, es el hecho de que el turista no utiliza servicios ni estructuras turísticas comerciales para la pernocta, sino que aprovecha una estructura habitacional (casa o departamento) que suele haber comprado o que renta fuera del sistema de alojamiento hotelero.

Una reciente definición aportada por Hiernaux (2005:3), establece que "El turismo de segundas residencias es aquel por el cual las personas acuden a 
un destino o una localidad que no es forzosamente turística per se, donde han adquirido la posesión por renta o compra de un inmueble en el cual pernoctan y realizan actividades de ocio y esparcimiento".

Una característica importante a señalar en la definición anterior, es que el destino en que se localiza la segunda residencia, no necesariamente debe ser turístico. Es decir, se puede dar el caso, por ejemplo, de una propiedad heredada localizada en el pueblo de origen de la familia paterna o materna y que se utiliza como residencia de descanso.

Tanto Herrera como Hiernaux, coinciden en los fines o propósitos por los cuales se adquiere una segunda residencia: descanso y recreación. Los turistas residenciales aprovechan la quietud y el clima del destino, para realizar diversas actividades, que entre ellas van desde leer hasta practicar deportes acuáticos.

De acuerdo a Herrera Romero (1997:44), el Turismo Residencial presenta tres rasgos particulares descritos a continuación:

a) El turismo residencial presenta un conjunto de relaciones y fenómenos originados por el desplazamiento; éstos pueden ser de tipo económico, social y físico.

En cuanto a las relaciones y fenómenos económicos se refiere, se tiene que cuando el turista realiza el desplazamiento, incluyendo trayecto y estancia, hace uso de servicios turísticos y no turísticos, como es el caso de tiendas de abarrotes, gasolineras o farmacias. La implementación de esta alternativa turística, dio paso a la generación de diversas fuentes de empleo, las cuales se caracterizan por ser de bajo nivel profesional, siendo una buena oportunidad laboral para los lugareños, quienes se 
desempeñan en quehaceres como vigilantes, empleadas domésticas, jardineros, entre otros. En estos empleos, no se requiere un elevado nivel de estudios.

Del mismo modo, al hacer los gastos de mantenimiento de la segunda residencia, como son: gas, luz, impuestos, entre otros, se contribuye con la economía de la región y no con los servicios turísticos.

Los turistas residenciales generalmente viajan en automóvil propio, debido a la frecuencia del desplazamiento, la distancia entre ambas residencias, y el tiempo de traslado. Hacer el viaje en transporte público implicaría más gasto y tiempo en completar el recorrido, además de ser más incómodo.

Los fenómenos de carácter social se suscitan cuando el turista residencial entra en contacto con la población de la comunidad receptora, a diferencia de los turistas tradicionales que hacen el contacto con prestadores de servicios, en especial turísticos.

Por último, los fenómenos de tipo físico se aprecian claramente con la presencia de éste tipo de turismo, ya que los inmuebles y en sí la urbanización, se ven modificados a consecuencia de la intervención de los turistas residenciales.

b) El desplazamiento voluntario y temporal. El turista residencial elige la frecuencia de los desplazamientos. Estos no siguen un patrón determinado de visitas; se hacen cada fin de semana, cada mes, en periodos vacacionales e incluso por suspensiones programadas por sucesión de días inhábiles, mejor conocidos como "puentes" o fines de semana largos, en los que regularmente se prefiere viajar con la familia y en ocasiones con parientes o amigos. 
c) Motivación de desplazamiento. La principal motivación del turista residencial es el descanso, ya que pretende alejarse de la monotonía o las presiones de las grandes ciudades; y la recreación, por ejemplo: actividades deportivas, sociales o de diversión nocturna, donde el turista residencial busca actividades diferentes a las que encuentra en la cotidianidad de la urbe en que habita.

Estos rasgos son la base de las características esenciales del turismo residencial, debido a que son invariables en cada caso de dicha modalidad de turismo.

A las anteriores características se añaden las siguientes con base en Hiernaux (2005):

d) El uso de una propiedad privada (fuera de la habitual) hace que el dueño adquiera nuevas responsabilidades, como por ejemplo: el mantenimiento de la misma y los pagos de impuestos, equivalente a lo que hace un administrador de un servicio de hospedaje tradicional. Esta tendencia provoca que exista una baja en la venta de cuartos hoteleros, cosa que los administradores ven a las inmobiliarias como competidores desleales.

e) Se sabe que el turismo residencial está sujeto a niveles económicos, debido a que para mantener una segunda residencia se necesita un alto poder adquisitivo. 


\section{REFERENCIA EMPÍRICA SOBRE MODELOS Y CARACTERÍSTICAS DEL TURISMO RESIDENCIAL EN MÉXICO}

De acuerdo a un estudio realizado por Hiernaux en el ámbito nacional en 15 destinos turísticos, el autor propone realizar una clasificación de las modalidades de turismo residencial identificadas en México con base en diferenciaciones regionales (2005:178) en las siguientes cuatro posibilidades:

a) Modelo de playa masificada a dominante nacional. La principal característica de este modelo es la existencia de fuertes atractivos locales, tanto playas como discotecas o restaurantes, los cuales pueden ser aprovechados de igual forma por los turistas tradicionales como los residenciales. Aquí se encuentran destinos como: Acapulco, Gro., Cancún, Q. Roo.

b) Modelo costero con fuerte presencia extranjera. Se distingue porque es mucho más mercantil que los demás: la necesidad de los turistas de adquirir una residencia preconstruida ha impulsado la presencia de la promoción inmobiliaria en forma creciente. Es evidente que los capitales extranjeros no se habían hecho presentes en la edificación de vivienda, y ahora lo hacen en forma masiva en destinos costeros como Puerto Peñasco. En estos casos se tiene gran participación inmobiliaria en los proyectos de desarrollo residencial, enfocado directamente a los extranjeros, debido a que, los precios están ya estipulados en dólares. El verano es la principal temporada de vacaciones que estos residentes secundarios aprovechan para el uso de sus casas de campo.

\section{c) Modelo de interior con atractivo turístico y dominante residencial.}

Se sabe que los turistas residenciales eligen el destino de acuerdo a ciertas características o atractivos del mismo, ya sean de tipo natural (Valle de Bravo - Ixtapan de la Sal) o cultural (Malinalco). La característica principal de este modelo es que existe poca oferta turística hotelera, en relación a la residencial. 
d) Modelo de interior masivo. Lo importante de este modelo es el hecho de que se ha consolidado, en el plazo de varias décadas, como una sombra de suburbanización turística de las grandes ciudades, como el Distrito Federal. Esto es, un proceso de desvanecimiento de la esfera residencial turístico que se presenta en un modelo de expansión indiscriminada y no controlada, donde se fusionan residencias principales, secundarias y viviendas de interés social.

El estado de Morelos es elegido por gran cantidad de turistas residenciales, ya que por su cercanía con el Distrito Federal, y su excelente clima, hace que la estancia sea más placentera, en comparación con una vida llena de stress y bullicio que se vive día a día en la gran ciudad. Debido a factores como la edad, tanto de los padres (tercera edad o jubilados), como de los hijos, esta segunda residencia puede convertirse a su vez en primera residencia.

A manera de síntesis se comenta que de acuerdo a cada destino, se nota una gran diferencia entre la oferta de segundas residencias y la oferta hotelera. Un ejemplo claro de ello es Mazatlán, que presenta altos índices de turismo tradicional; contrario a ello, se tiene que Valle de Bravo supera el número de segundas residencias al de hoteles.

Algo importante a recalcar que atinadamente menciona Hiernaux (2005:103), es el desconocimiento de la oferta que se tiene en todos los municipios, ya que resulta casi imposible obtener datos de las autoridades locales, sean o no relacionadas a la actividad turística. Lo que demuestra que este segmento está totalmente fuera de los intereses de conocimiento de las autoridades.

Como ya se había mencionado, la demanda de la segunda residencia está caracterizadaporgentecon un elevadonivel socioeconómico. Estajustificación "elitista" se da en función de que detrás de la segunda residencia existen 
gastos que el nivel económico promedio no puede costear. Estos consumos van desde la adquisición de la vivienda hasta el mantenimiento periódico de la misma, por lo que se identifican dos tipos de egreso: a) mantenimiento básico, es decir limpieza, jardinería y conservación de la vivienda para poder ocuparla con regularidad, a lo que hay que agregar el costo de los servicios municipales; y b) mantenimientos mayores en reparaciones.

En cuanto a demanda internacional se refiere, se tiene que el país vecino del norte, ha mostrado mucho interés en las costas mexicanas, específicamente de Baja California Norte y Sur, así como una porción de Sonora. Esto es debido a que sus inversiones son bien vistas por las numerosas inmobiliarias que se han desarrollado en dicha región costera. Dentro de esta población de residentes, están los que tienen una estacionalidad de más de tres meses, generalmente ciudadanos americanos de la tercera edad, esto representa a su vez, un contingente importante de inmigrantes temporales.

Se puede agregar que el gasto turístico realizado por los turistas residenciales es inferior al que se realiza de modo tradicional, ya que en el fenómeno residencial la tendencia es que se suelen traer consigo los bienes elementales que se requieren para la estancia. Esto no es muy frecuente en los centros turísticos de playa, toda vez que es en éstos donde se acude con más frecuencia a lugares de esparcimiento y/o restaurantes.

El interés de una segunda residencia radica en la elección adecuada del destino, por lo que las razones deben satisfacer a los futuros dueños. La principal razón para ello es la calidad del clima y del lugar en sí. Sin embargo, la presencia de parientes o amigos (y las ganas de estar cerca de ellos) en el destino, también es una buena justificación para la adquisición de una propiedad. 
En forma similar actúan las motivaciones por las cuales se acude al destino. En una primera instancia se tiene que los paseos, deportes, salud, el acceso a la vida nocturna, playas y mares, son los principales motivos por los que se da la inclinación por una segunda residencia.

Sumados a los anteriores, se tienen aspectos como la tranquilidad, la convivencia familiar o con amigos, las condiciones de pazy descanso, también solicitados por estos turistas. Cabe señalar que esto depende también de los atractivos de cada lugar, debido a que estos aspectos son característicos de destinos del interior. 


\section{ASPECTOS TURÍSTICOS DEL MUNICIPIO DE IXTAPAN DE LA SAL}

Los servicios turísticos de Ixtapan de la Sal están conformados por aquellos que tienen que ver con hospedaje de diferentes categorías, alimentación, transporte interno y externo e instalaciones para recreación y esparcimiento como discotecas, salones para fiestas, venta de artesanías, los balnearios como centros de salud y belleza y facilidades para practicar deportes como la natación, equitación, golf y pesca deportiva.

Hay que mencionar que Ixtapan de la Sal originalmente se impulsó turísticamente como un destino de salud, por esto la oferta de servicios más atractiva y más conocida es, precisamente, la que está asociada a este segmento. Sin embargo, actualmente existe una preocupación por parte de la administración municipal y de los prestadores de servicios en aumentar y reorientar la oferta turística de la ciudad (Ricaurte;2001:8).

Con respecto a la planta hotelera, Ixtapan de la Sal cuenta con tres hoteles de cinco estrellas que son el Hotel Ixtapan, el Hotel Rancho San Diego y el Hotel Marriot Ixtapan (antes Hotel del Rey). Son precisamente estos tres los que poseen la mayor cantidad de instalaciones para realizar diferentes actividades recreativas y constituyen verdaderos puntos de concentración de la oferta turística de Ixtapan de la Sal.

Es importante señalar que la nueva Ley de Turismo desregula las tarifas de los establecimientos de hospedaje y alimentación de acuerdo a su clasificación por estrellas; de igual forma, ya no es obligatorio el registro de dichos establecimientos en la Dirección de Turismo del Estado que ha asumido un papel de supervisión y apoyo a la actividad, en lugar de controlarla. Así, al no contar con un organismo de control, se convierte en una dificultad para el gobierno municipal tratar de regularizar los establecimientos de alojamiento y peor aún, cobrarles el "impuesto al hospedaje" que consiste en el $3 \%$ de sus ganancias (Ricaurte;2001:9). 
En el municipio y específicamente en el Centro de Población Estratégico de Ixtapan de Sal, existe un mercado consolidado de bienes inmuebles, ya que la oferta consiste en los lotes de los desarrollos Rancho San Diego, Club de Golf Ixtapan Country, Ixtamil, Los Cipreses, etc. y los que individualmente se ofertan. La mayoría de estos se ubican dentro de la cabecera municipal o en su periferia inmediata, debido a la situación general de la economía nacional (GEM;2003:48).

A nivel general existen áreas urbanizables e incluso baldíos para que se aumente tanto la población local o inmigrante, como los visitantes de fin de semana. 


\section{ESTRUCTURA URBANA DE IXTAPAN DE LA SAL}

De acuerdo al Plan de Desarrollo Urbano (2003:66), el Centro de Población Estratégico se encuentra estructurado a partir del boulevard San Román, el cual ha conformado un corredor turístico y de servicios.

El uso de suelo en el Centro de la Población, es en su mayoría habitacional tipo residencial y popular medio, con porcentajes muy bajos de jardines y de áreas de recreación públicos; los servicios turísticos y desarrollos campestres tienen un área semejante a la vivienda tradicional, definiendo claramente la función económica de este centro.

La estructura urbana de Ixtapan de la Sal tiene como elementos básicos al "centro" de la localidad, los equipamientos públicos municipales, el balneario de Nueva Ixtapan, la avenida Benito Juárez (principal corredor urbano). Esta une a los centros mencionados y el boulevard San Román, como trayecto urbano de la carretera 55 que va a Tonatico y posteriormente a Taxco, Gro., liga a la mayoría de los sectores de la localidad (GEM;2003:105).

Según el Plan Municipal de Desarrollo Urbano de Ixtapan de la Sal (2003:54), el Boulevard Ixtapan - Tonatico se constituye en el principal integrador de la estructura urbana de la cabecera municipal de Ixtapan de la Sal y de ésta con la de Tonatico, conformando un Corredor Urbano Turístico que tiene una longitud de $6.12 \mathrm{kms}$. Inicia al norte del área urbana, en donde entronca con la autopista de cuota y la carretera libre, que viene de Toluca y continúa hacia el poniente y el sur, hasta la colindancia con el municipio de Tonatico y se desarrolla en cuatro tramos: 
Plano No. 1

Boulevard Ixtapan de la Sal - Tonatico

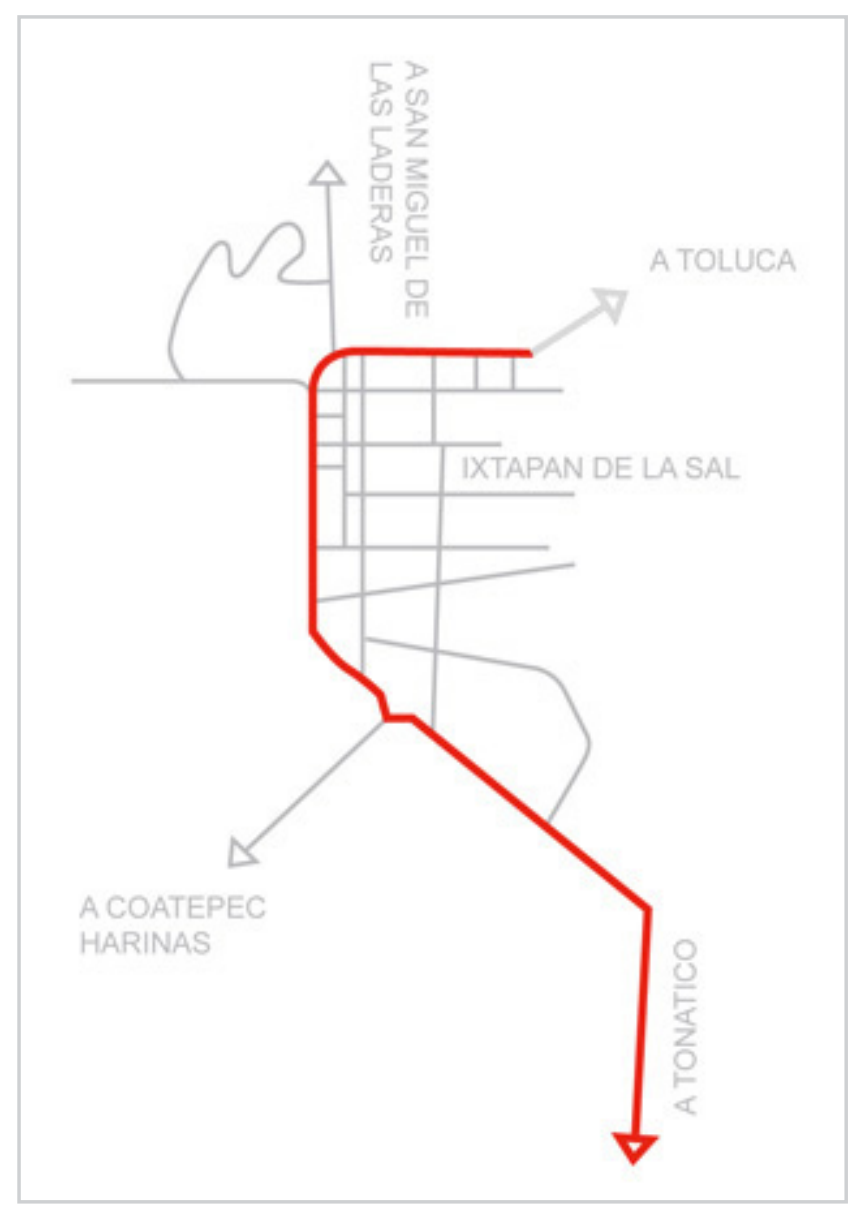

FUENTE: Secretaría de Desarrollo Urbano y Vivienda, Ixtapan de la Sal (2005). 


\section{EL TURISMO RESIDENCIAL EN LA CABECERA MUNICIPAL DE IXTAPAN DE LA SAL}

En el caso de Ixtapan de la Sal, se tiene que fueron las aguas termales las que propiciaron inversiones con fines turísticos, iniciándose la oferta de alojamiento tradicional, para posteriormente iniciar la residencial, gracias a las ventajas climáticas con las que cuenta el destino. Dicha oferta es menor que la hotelera, pero está aún en progreso, el proceso se da de manera individual, la venta se hace por lotes y es aquí donde actúan los promotores inmobiliarios.

Las zonas de asentamiento de los conjuntos inmobiliarios de segundas residencias se desarrollaron conforme al patrón seguido por el desarrollo turístico, esto es, el primer conjunto de casas se instalaron cerca del balneario y el Hotel Ixtapan, sobre el boulevard San Román, seguido de las casas construidas en la colonia Bugambilias, alrededor de los años ochentas. Fue al principio de la década de los años noventas cuando en la zona noreste se instaló el Ixtapan Golf Resort \& Country Club (conjunto inmobiliario de la familia San Román), y en el sureste los fraccionamientos de El Ciprés, Xochicaltitlán y Rancho San Diego (antigua propiedad del Rancho Las Animas), este último instalándose en una pendiente del cerro que proporciona una vista panorámica sobre la cabecera municipal (Osorio;2004:6).

De acuerdo al censo de segundas residencias realizado en la cabecera de Ixtapan de la Sal, hasta el mes de octubre de 2005, existen alrededor de 265 casas de fin de semana. Esta estimación es calculada, sin contar con el fraccionamiento "La Joya", donde se cree que hay diez casas residenciales, ni con el nuevo complejo aún en construcción, "Residencial Ixtapan" (colindante con el Ixtapan Golf Resort \& Country Club). Este último consta de tres secciones: popular, habitacional y residencial; desconociéndose datos de los dueños, procedencia y permanencia. A continuación se presentan los fraccionamientos identificados como espacios de ejercicio de turismo residencial en la cabecera de municipal de Ixtapan de la Sal: 
Plano No. 2. Ubicación de segundas residencias de la cabecera municipal de Ixtapan de la Sal

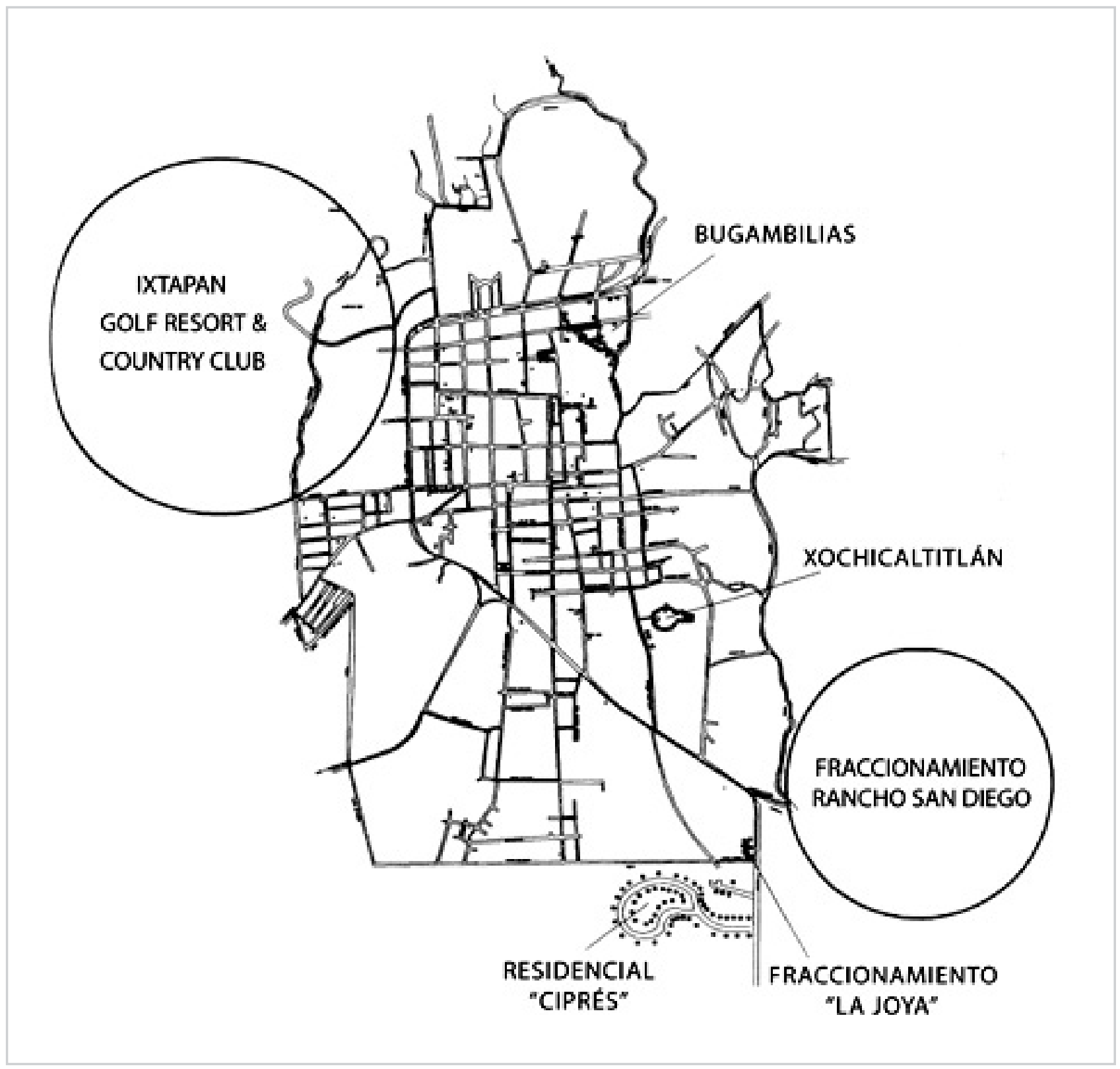

FUENTE: INEGI (2000). 
a) Colonia Bugambilias. Es un conjunto habitacional de casas solas de nivel medio y residencial cuyo auge se registró durante la década de los años ochentas. No se encuentra cercado y se ha constituido como una colonia más de la población. Algunas de éstas casas están en venta o en renta, debido a que permanecen abandonadas, por lo general son construcciones viejas, se puede decir que fueron de las primeras casas de fin de semana de Ixtapan de la Sal.

b) Fraccionamiento Residencial Ciprés. Es un conjunto habitacional cercado constituido de 53 casas solas clasificadas como residenciales. Los lotes van de los 600 a los 1,000 m2. No cuenta con servicio de agua potable, para ello utilizan la recolección pluvial. El comprador adquiere un lote que construye a su gusto, pero siguiendo especificaciones de construcción (estilo campestre). Los dueños formaron un comité para tomar acuerdos sobre los pagos que deben realizar en conjunto, tal es el caso del salario de los empleados. Los pagos de impuestos y de luz, los realiza tanto el dueño como el administrador del fraccionamiento.

c) Fraccionamiento Xochicaltitlán. Es un conjunto habitacional de 24 casas solas de nivel medio, cercado. Sus residentes fueron adquiriendo los lotes para construir bajo su propio gusto, sin someterse a especificaciones de construcción. Ellos se hacen cargo de los pagos de impuestos, agua, luz, etc.

d) Fraccionamiento Rancho San Diego. Su autorización se remonta a 1993, aunque a raíz de la crisis de 1994 su despegue se llevó a cabo de manera muy lenta, reactivándose a partir de 1998. El terreno se extiende en el territorio del municipio de Ixtapan de la Sal abarcando 250 hectáreas sobre la pendiente del cerro que se localiza al sureste de la cabecera, no obstante se ha continuado la compra de terrenos aledaños por lo que se 
estima que ha aumentado su extensión. De acuerdo al plan original, el fraccionamiento se creó como un "centro turístico recreativo", aunque se sabe que desea transformarse en "conjunto urbano de vivienda popular". La población prevista para este fraccionamiento corresponde a 1,198 lotes, 28 manzanas y 3,529 viviendas, lo que a razón de 4.6 habitantes por vivienda implicará una población de 16,870 habitantes. El fraccionamiento cuenta con agua propia, esto consiste en pozos de agua notariados, los cuales alcanzan para el abasto de dos mil casas con alberca. La situación es preocupante en tanto que la zona no cuenta con drenaje y los desechos son descargados en fosas sépticas con digestión anaerobia, es decir, dichas fosas son conectadas al drenaje de la calle.

Para el año 2005 existían 70 casas construidas y 10 en construcción, lo que muestra un problema de venta en comparación con las ventas programadas. Se tienen 2200 lotes y se venden dos terrenos por cliente. Estos lotes van de los $400 \mathrm{~m} 2$ en adelante y se distinguen por tres niveles: especiales (con vista panorámica); medios (toda el área); y normales (son los más económicos y se localizan en la ladera o barranca). El comprador adquiere un lote y construye con ciertas especificaciones de estilo mexicano contemporáneo, los servicios deben estar ocultos, tener $9 \mathrm{mts}$., de banqueta hacia arriba, bien sea, en dos o tres niveles. Los pagos de impuestos y de luz los realiza tanto el dueño como el administrador del fraccionamiento. Se conoce que actualmente hay propietarios extranjeros de algunos lotes.

e) Fraccionamiento Ixtapan Golf \& Country Club. Propiedad de la familia San Román, el fraccionamiento creado en 1998, es un conjunto cerrado con 300 has, que cuenta con todos los servicios públicos, sin problema de abastecimiento de agua y de alto lujo. 
Los servicios que anuncia su página de internet son: a) campo profesional de golf con 18+1 hoyos, tee de práctica y putting green, b) lotes residenciales, con servicios urbanos ultra modernos, c) hermosas y funcionales villas, d) casa club con spa de clase mundial, e) salones para usos múltiples, f) instalaciones profesionales para la práctica de tenis, padel tenis, natación y actividades ecuestres, g) ciclismo de montaña, h) kids Club, i) lago para pesca deportiva, j) lago para remar, k) salón de juegos, y I) escuela bilingüe reconocida, con enseñanza pre-escolar y primaria.

Para el caso del fraccionamiento, se presentan tres alternativas de ventas: 1) terrenos en fairway, que colindan con el campo de golf, 2) terrenos panorámicos o campestres, con vistas de todo el desarrollo y 3) villas con un diseño vanguardista y acabados de primera calidad.

La venta de los terrenos se realiza en dólares y se divide en los colindantes con el campo de golf; los panorámicos o campestres; y los de interés social. Los primeros son los de mayor lujo y exclusividad, teniendo una localización altamente favorable. Los segundos se destinan a un nivel medio alto y los terceros están considerados como casas habitación para trabajadores de la empresa. Como servicios exclusivos de la zona residencial, se anuncian los siguientes: a) Presas de almacenamiento, para suministro de agua potable, con capacidad para 250,000 m3, b) Planta potabilizadora de agua, c) Sistemas de alta seguridad, alumbrado público especial, barda perimetral y vigilancia las 24 horas del día, d) Líneas telefónicas, e) Servicios generales de mantenimiento, como plomería, electricidad y jardinería (con costo adicional), f) Sistemas hidráulicos y eléctricos a pie de lote, y g) Centro comercial.

Como se puede observar, su condición es de ventaja con relación al suministro de agua y a las garantías de seguridad. 
Existen 46 casas construidas en el complejo residencial (año 2005), sin embargo, se tiene proyectado edificar un total de 600 casas. El costo de cada terreno es de un millón y medio de pesos, más 30 mil dólares de membresía (obligatorio), más 2 mil pesos mensuales de mantenimiento. Si el dueño desea tener una partida de golf le cuesta 2 mil pesos el green fee por día.

El pago de la residencia debe ser al contado, y al comprador se le otorgan seis meses para construir la propiedad, de lo contrario se pierde el contrato. El administrador ofrece 5 diferentes modelos de residencia, de los cuales el dueño escoge a su gusto, dichos modelos van de los 2 a los 6 millones de pesos.

Como se puede apreciar, la cabecera municipal tiene zonas claramente identificadas para uso exclusivo de turismo residencial, las cuales en su mayoría se encuentran en el corredor turístico Ixtapan - Tonatico.

En cuanto al perfil del turista residencial se puede afirmar que la principal entidad emisora de turistas residenciales es el Estado de México, particularmente la capital mexiquense, municipio de Metepec y Distrito Federal, debido a la cercanía de dichas metrópolis con Ixtapan de la Sal, ya que el tiempo de recorrido es de menos de tres horas.

Generalmente acuden familias de cuatro integrantes, y el gasto turístico que realizan dentro de la ciudad es mínimo, debido a que únicamente compran alimentos para prepararlos en su vivienda, sin la necesidad de asistir a restaurantes. En ocasiones optan por dar paseos nocturnos en el centro de la ciudad, puesto que recientemente se empezó a ofrecer un espectáculo de luz y sonido en la fuente del jardín principal, lo cual atrajo muchos espectadores tanto residentes como ixtapenses. 
El número de visitas es de uno a dos desplazamientos por mes, lo que significa que son muchas las visitas al año, por lo tanto se muestra un recorrido periódico hacia la segunda residencia. Aprovechando la frecuencia de visitas, los residentes gustan de acudir a darse un baño en las aguas termales 0 prefieren atenderse en el Spa.

Las agencias inmobiliarias han jugado un papel importante dentro del desarrollo de la vivienda de lujo, ya que es mediante éstas que el auge residencial ha ido en aumento, y el mayor número de adquisiciones de casas se ha dado por esta vía.

De acuerdo con los modelos propuestos por Hiernaux (2005), se tiene que Ixtapan de la Sal, caracterizado como destino del interior con atractivo turístico, aún no supera la oferta hotelera como es el caso de Valle de Bravo o Tequisquiapan, Qro. Sin embargo, la promoción de las segundas residencias va en aumento. 


\section{IMPACTOS DEL TURISMO RESIDENCIAL}

Con base en el trabajo realizado por el Dr. Hiernaux (2005), los impactos estimados para el turismo de segundas residencias de Ixtapan de la Sal están vinculados con las siguientes variables:

1) Estructura urbana. Este impacto es uno de los más notorios, ya que por el tipo de construcciones, es fácil encontrar albercas o grandes jardines dentro de los conjuntos residenciales, caso opuesto a las primeras residencias donde la edificación de la propiedad es modesta.

Estos cambios originados por el desarrollo de la actividad turística, están transformando su paisaje natural a otro en donde el hombre ha intervenido, descuidando la imagen, como es el caso de la contaminación de arroyos. La adquisición y construcción de conjuntos residenciales en cerros vecinos a la cabecera municipal ha alterado la imagen original de Ixtapan de la Sal, ya que el uso agrícola del suelo se está transformando paulatinamente a uno habitacional, específicamente de vivienda de lujo de estilo campestre.

El paisaje urbano es el primer indicio de cambio de estructura urbana de cualquier localidad en el proceso de desarrollo, debido a la aparición a simple vista de servicios y complejos turísticos, y en este caso, complejos residenciales, los cuales son claramente identificados por sus singulares construcciones.

Aunado a lo anterior, se tiene el Residencial Ixtapan (nuevo conjunto residencial) en donde cada lote estará urbanizado, ya que dentro del costo se incluye además de los servicios comunes, drenaje pluvial y cableado subterráneo.

La Secretaría de Desarrollo Urbano y Vivienda del municipio de Ixtapan 
de la Sal (2003), ha examinado una serie de directrices o tendencias, con la finalidad de analizar la dinámica urbana y conocer su problemática sin afectar el medio urbano.

Se tienen tendencias, las cuales están siendo asimiladas para que el desarrollo urbano tenga un avance constante, sin alterar la estructura urbana y la función de la misma.

Dado que sólo la cabecera municipal tiene un índice demográfico importante $(15,856$ habitantes para el año 2003) y que es la única localidad con un patrón de asentamiento consolidado, es de esperarse que el crecimiento con características "urbanas" se dé casi exclusivamente en ella, aunque los pueblos de San Alejo $(1,111)$, Ahuacatitlán $(1,353)$ y Tecomatepec $(1,633)$ pudieran compartir una proporción considerable. Las otras localidades son aún muy pequeñas y seguirán correspondiendo al ámbito rural (GEM;2003:75).

Al sureste, seguirá edificándose en el Rancho San Diego. Aunque hay que considerar que este desarrollo está autorizado como "conjunto urbano", tiene como destino el turismo de fin de semana, y si con el tiempo algunas personas llegaran a residir en este fraccionamiento como ha sucedido en Valle de Bravo, se estima que esta proporción no rebase el 15\% de su capacidad, por lo que su impacto local, a corto y mediano plazos en el Centro de Población, se prevé de bajo nivel. Se debe recordar que parte de este conjunto se ubica en Tonatico por lo cual se ha contribuido al proceso de conurbación intermunicipal que se reporta como característico en la subregión.

Hacia el sureste del boulevard San Román, la urbanización se está incrementando, y así continuará con rumbo hacia Tecomatepec donde 
ya se pueden apreciar nuevas edificaciones, algunas de las cuales se localizan por arriba de la cota que se pretende manejar como límite de crecimiento urbano, por lo que se debe realizar una supervisión que evite su proliferación.

En este sector, la tendencia es a expandirse, dadas las escasas pendientes que se presentan en la planicie existente y en el lomerío suave del sur. Ejemplo de ello son las colonias Lindavista y La Joya, donde se aprecian nuevas construcciones que se deben controlar.

Una de las consecuencias originadas por dichos complejos, es el aumento de precio del valor catastral de los terrenos. La Coordinación de Catastro Municipal designa los costos de los terrenos, estableciendo una codificación de las áreas homogéneas de acuerdo al uso del suelo y niveles de jerarquización, de los cuales el que interesa para fines de este estudio, es el Habitacional $(\mathrm{H})$, destinado a la vivienda y resguardo de la población (Catastro;2005:689). Esta clasificación a su vez se divide en categorías que van desde $\mathrm{H} 1$ hasta $\mathrm{H} 6$, la última hace referencia a zonas altamente exclusivas, normalmente fuera de las zonas consolidadas; y $\mathrm{H} 1$, que es para las periferias de las ciudades y/o pueblos, con un régimen jurídico de la propiedad catalogado como ejidal, con costos que van desde los $\$ 50.00$ por metro cuadrado. En Ixtapan de la Sal la mayor categoría reconocida es $\mathrm{H} 4$, registrada como privada, con un costo que va de los $\$ 500.00$ hasta los $\$ 1,300.00$ por metro cuadrado (Country Club). Es así que a medida que incrementan los servicios dentro y alrededor de los predios, el importe será aún mayor que para aquellos adquiridos sin servicios.

La infraestructura urbana, así como los servicios, son cómplices de estos impactos, ya que sin ellos, la simple edificación de residencias no sería nada. 
2) Servicios urbanos. Estos, a modo de impactos ambientales influyen en factores como contaminación de aguas, o bien, en mejoras en la calidad ambiental, diversidad en el uso de terreno y modificaciones en la estructura urbana, describiéndose a continuación:

En cuanto a la contaminación del agua, se tiene que en Ixtapan de la Sal, el Sistema de Alcantarillado contaba para el 2003 con una cobertura de 5,129 descargas de aguas residuales, equivalente al $76.4 \%$ del total de viviendas registradas en el municipio, beneficiando a 23,000 habitantes aproximadamente, lo que expresa un rezago de 1,584 viviendas que no cuentan con servicio de alcantarillado (GEM;2003:51).

Este es uno de los rubros con mayor carencia, al igual que las redes de agua potable, que corren en sentido norte-sur siguiendo la pendiente natural del terreno.

El sistema de drenaje municipal tiene como eje de desagüe, un canal a cielo abierto denominado arroyo El Salado, ubicado al poniente del área urbana.

Desde que se inició la construcción de Rancho San Diego no funciona la laguna de oxidación localizada al sur del área urbana, por lo que las aguas residuales que allíse captaban y trataban son vertidas indiscriminadamente al Río Salado.

La contaminación del agua proviene de las descargas de las viviendas y los comercios, además de las descargas producidas en los establecimientos de servicios turísticos, tales como hoteles y balnearios. 
De acuerdo a las entrevistas realizadas y a los documentos consultados, se identifica que los impactos generados por los fraccionamientos de segundas residencias en el ambiente incluyen un incremento en el consumo del agua y su disponibilidad negativa a mediano plazo para la población local, asimismo se observa el crecimiento del volumen de los desechos sólidos y la posibilidad de contaminación acelerada.

Existe un grave problema con el abastecimiento de agua en el municipio, ya que se está incrementando su consumo. Para ello, el Organismo Descentralizado para la prestación de los Servicios de Agua Potable, Alcantarillado y Saneamiento (ODAPAS) ha pedido ampliar la concesión de agua de $15 \mathrm{lts} / \mathrm{s}$ que se tiene actualmente. Sin embargo, el municipio necesita 60 lts/s, aún cuando dentro de este abasto de agua no entra el fraccionamiento residencial Rancho San Diego, debido a que cuenta con pozos de agua, lo que lo hace independiente del servicio.

No obstante se está desarrollando una mejora en la calidad ambiental, debido a que el funcionamiento de la red de agua en el centro de población tiene un $80 \%$ de eficiencia, considerando que es necesario resolver el problema de algunos cruceros conflictivos en el drenaje, especialmente en época de lluvias. Por ello se requiere renovar las líneas de mayor problemática; recanalizar los cruceros conflictivos; y reestructurar la zona de descarga de aguas residuales hacia un punto estratégico, además de independizar la conducción de aguas negras de las pluviales.

Sumado a lo anterior se dice que gran parte de la infraestructura hidráulica existente presenta deficiencia por falta de mantenimiento y por la antigüedad de la misma, por lo que es necesario realizar obras de mantenimiento, rehabilitación y ampliación para prevenir problemas de contaminación. Actualmente se están cambiando las pequeñas tuberías 
instaladas en un principio por otras de mayor captación, las cuales darán mejor desahogo en la red de tuberías.

Por otra parte, existen políticas que servirán para dar soluciones o prevención a estos impactos, los cuales se describen a continuación (GEM;2003:131):

- Impulsar la construcción de plantas de tratamiento de aguas residuales para reusarlas de acuerdo a la normatividad vigente.

- Impulsar la realización del proyecto de colectores de aguas servidas, sobre todo aquellos paralelos a los cauces de arroyos que son utilizados como drenajes.

- Llevar a cabo las acciones de mantenimiento preventivo y correctivo de la red.

- Apoyar la realización del desazolve de las alcantarillas y causes.

EI ODAPAS menciona que el presupuesto anual no es suficiente para la realización de las anteriores políticas anteriormente descritas, a excepción de la última, debido a que la maquinaria utilizada para desazolvar las alcantarillas se pide prestada periódicamente a municipios grandes como Toluca, Metepec o Ecatepec.

El turismo residencial se verá beneficiado con estas políticas de mejora de líneas de agua (alcantarillado), gracias a que en la red de drenaje de algunos de los fraccionamientos (Rancho San Diego) que actualmente funcionan con fosas sépticas, se tiene la opción de enlazarse al sistema de alcantarillado del municipio. Este sistema se denomina "fosa séptica con digestión anaerobia".

Los equipamientos de apoyo al turismo se encuentran distribuidos en el 
centro, norte y sur de la cabecera y están constituidos fundamentalmente por hoteles, casas de huéspedes y nuevos desarrollos con viviendas para fin de semana.

Por otro lado, Ixtapan de la Sal ha mostrado buena cara al turismo residencial y lo que ha traído con él, debido a que se está estimulando la generación de la urbanización del espacio.

Se está cambiando la estructura urbana de la cabecera, debido a que con el auge de fraccionamientos enfocados a las segundas residencias, la ocupación de suelos está cambiando su camino, ya que provoca un aumento en la gama de servicios (redes de agua, luz, pavimentación, etc.) dentro de la zona, acarreando consigo un beneficio para la comunidad local.

Los fraccionadores sacan partido de comprar terrenos a precios muy bajos. Una vez dividido el terreno y agregados los servicios necesarios de vivienda, estos predios construidos han propiciado un marcado encarecimiento del suelo.

Como se dijo anteriormente, gran parte de la vivienda para uso habitacional es destinada al turismo residencial, por lo que grandes porciones de tierra son fraccionadas para la comercialización de las segundas residencias.

3) Incremento en el mercado laboral del destino. La importancia del turismo como fuente generadora de empleos está íntimamente ligada al grado de importancia que aquél revista como fuente generadora de ingresos para el país. Por esta causa, en aquellos países donde el producto interno producido por el turismo es relativamente bajo, el empleo en este "sector" generalmente representa una pequeña proporción del empleo 
total. En cambio, en los países donde el turismo tiene una gran significación como fuente generadora de ingresos, como es el caso concreto de muchos países de la región del Caribe, dicha actividad aumenta su importancia como fuente generadora de empleos (Acerenza;1991:106).

A partir del aprovechamiento comercial de los recursos naturales (aguas termales) de Ixtapan de la Sal, empezaron a desarrollarse gran variedad de establecimientos dedicados a comercializar productos llamativos para el visitante de las aguas termales, así como la creación de nuevos empleos, enfocados al bienestar del turista.

Si bien es sabido que el turismo tradicional es un motor generador de empleo, el turismo de segundas residencias no se queda atrás. Este también genera empleos directos, aunque no son de elevado nivel profesional, debido a que las tareas del personal son servicio doméstico, jardinería o mantenimiento. Dichos oficios o tareas no exigen gran preparación académica o capacitación, pero sin éstos, los mismos dueños tendrían que encargarse del mantenimiento o desperfectos de la vivienda.

De acuerdo a los resultados de la investigación de campo, se pudo conocer que los empleos de vigilancia o seguridad son muy usuales en Ixtapan de la Sal, debido a que existen varios fraccionamientos que requieren de personas que se encarguen de estas obligaciones, teniendo así un ingreso promedio mensual fijo de $\$ 3,700$, del cual la mitad de los trabajadores aportan más del $50 \%$ al gasto familiar.

Una cuarta parte de los encuestados afirmó haber trabajado anteriormente en algo relacionado en el turismo; a su vez, todos los empleados aseguraron haber trabajado en el campo, lo que indica, como se mencionó anteriormente, que se está cambiando el uso de la tierra y con 
ello la ocupación en el empleo. Los trabajadores de segundas residencias no muestran tener ventajas o desventajas con la frecuencia de visita de los dueños, debido a que el turista residencial llegue o no a su casa, el trabajador siempre tiene que estar ahí, pero es importante mencionar que aseguraron tener buenas relaciones con los propietarios.

El empleo indirecto se refiere a los trabajos relacionados con otros sectores de la economía vinculados con el turismo, los cuales no siempre están en contacto directo con el turista, sin embargo, auxilian al visitante y hacen que la estancia sea más cómoda, ejemplos de estos tipos de empleos son: tenderos, conductores de transporte público, empleado de mostrador de farmacias o despachadores de combustible, que para el turista residencial son más útiles que los servicios turísticos tradicionales.

La generación de empleos no sólo ayuda al funcionamiento de los fraccionamientos, sino también a la manutención de muchas familias, que se ven beneficiadas por estas oportunidades de tener un trabajo, a pesar de que en algunos casos no se cuenta con la experiencia profesional para conseguir un empleo con más exigencias académicas o de capacitación para el trabajo.

4) Implantación de comercios especializados. Además de los empleos, se debe mencionar la diversificación económica que ha acarreado la actividad turística, y los cambios mismos que han ocurrido en ella.

Se ha abierto una gran gama de posibilidades en cuanto a la implantación de comercios especializados para la atención del mercado de segundas residencias, por ejemplo los establecimientos de mantenimiento de albercas o casas de materiales con insumos de lujo. Así, se constituye progresivamente un segmento de la economía local que se sustenta 
en el turismo de segunda residencia como una nueva demanda, con características particulares adecuadas al tipo de actividades realizadas por este tipo de turistas (Hiernaux; 2005: 22).

Para contar con un conocimiento actualizado sobre la situación de los servicios turísticos se llevó a cabo un sondeo entre los prestadores de servicios y comerciantes diversos, estos últimos con el interés de conocer la experiencia con los turistas tradicionales y residenciales. Los resultados refieren que la mayoría de los comerciantes encuestados opinó que su negocio es rentable, debido a que además de atender las necesidades de la comunidad receptora, se encargan de los turistas tradicionales.

Existen varios destinos en la República Mexicana que cuentan con la misma actividad residencial, pero el caso específico de Ixtapan de la Sal está luchando por un lugar significativo dentro de la preferencia del turista, sobre destinos residenciales como Acapulco, Valle de Bravo o el estado de Morelos.

El turismo residencial como reciente y creciente actividad turística en Ixtapan de la Sal, está tomando una fuerte atracción por el potencial turista de segundas residencias, debido a sus ventajas sobre las que tiene al alojarse en un hotel. 


\section{CONCLUSIONES}

A través de la exposición se han visto elementos importantes para adentrar al lector a un tipo de turismo incomprendido: el "turismo residencial", del cual, a través de diferentes apartados se pudo conocer uno de los casos en el Estado de México, Ixtapan de la Sal. Este lugar conserva su tendencia a atraer turismo doméstico, con la diferencia de que no hace uso de los tradicionales servicios que presta dicho destino.

El tipo de clima con que cuenta el municipio es favorable para el desarrollo de las segundas residencias, siendo una ventaja para aquellos dueños que radican en localidades menos privilegiadas climáticamente como son los casos del Distrito Federal y Toluca.

No obstante que en Ixtapan de la Sal hay un mayor número de cuartos de hotel que de segundas residencias, éste segmento va en ascenso. El turismo residencial de Ixtapan de la Sal se desarrolla a partir de un esquema inmobiliario en el que prevalecen los fraccionamientos. Es decir, no responde a una transacción directa de vendedor a comprador por casa sola, sino de un negocio de mayor escala sobre la lotificación de terrenos para su venta a varios compradores. Esto revela una tendencia de mercado creciente sobre la compra-venta de terrenos para vivienda para visitantes externos destinada al descanso.

Las reglas de construcción y vivienda rigen la calidad y seguridad de la construcción de las residencias, además de su posterior mantenimiento. En la mayoría de los casos, estas normativas impuestas por los fraccionadores especifican los materiales que se han de utilizar, su calidad mínima y los componentes de construcción con los que debe contar una estructura adecuada para la ocupación humana, además de no alterar el paisaje, tratando de mantener un estilo campestre. 
El perfil del turista residencial presenta características muy marcadas, en comparación con las del turismo tradicional. Por ejemplo, el turista de segundas residencias no hace uso de servicios de alojamiento, ya que la estancia es realizada en una casa que por lo general es propia. El automóvil es el principal transporte utilizado por estos turistas residenciales.

Durante la investigación se constató que la posición económica es un factor importante para poder entrar al gremio de los turistas residenciales, ya que se debe tener un elevado nivel económico para poder adquirir y dar mantenimiento a una casa adicional.

Esto hace que el turismo residencial se vea como una posibilidad de elevar el status económicamente hablando, debido a que la situación financiera limita a ciertos grupos y privilegia a otros, siendo así un turismo elitista.

La adquisición de grandes porciones de terreno por parte de capitalistas para hacer fraccionamientos residenciales, obliga a la comunidad receptora de menos posibilidades económicas a mudarse a las orillas de la Cabecera Municipal.

El auge de este tipo de turismo ha traído consigo una serie de repercusiones, tanto ambientales-urbanas, como económicas. Dentro de las ambientales - urbanas, se tiene que se ha modificado el paisaje natural por la creación de conjuntos residenciales, la contaminación de ríos y arroyos. Esta es provocada por desechos de servicios turísticos, comercios y viviendas principalmente lo cual, por falta de recursos económicos, no se han podido resolver, a pesar de que se tienen los proyectos para ello.

Por la parte económica, se tiene que el turismo de segundas residencias trae consigo cambios. La generación de empleo (de bajo nivel profesional) 
es uno de ellos, ya que estas casas necesitan de personas dedicadas a la construcción y mantenimiento -llámese jardinería, servicio doméstico, etc.- y debido a que hay varios fraccionamientos, se necesita vigilancia y seguridad de las propiedades, ya que algunas de estas residencias pertenecen a figuras públicas o con alto poder adquisitivo.

Por último, el turismo residencial es otra opción para atraer turistas, sin importar que el destino tenga vocación turística, pero que cuente con al menos un atractivo que llame la atención del visitante para que lo motive a adquirir una residencia adicional a la habitual. Además, el aumento de desarrollos residenciales en el lugar, beneficia a la comunidad receptora y hace que el turista residencial, se sienta "como en casa".

De esta forma se puede comprender un poco acerca del comportamiento del turista residencial, en un entorno completamente distinto al que habita la mayor parte de su vida, dando paso al ocio el cual es una necesidad humana, originado por el dinámico ritmo de vida citadino, es aquí donde se recarga la capacidad de trabajar nuevamente. 


\section{BIBLIOGRAFÍA}

- Acerenza, Miguel Angel (1991), Administración del turismo.

- Coordinación de Catastro Municipal (2005), Reglamento del titulo quinto del código financiero del Estado de México y municipios denominado "Del Catastro".

- GEM (2003), Plan Municipal de Desarrollo Urbano de Ixtapan de la Sal. Toluca: Secretaría de Desarrollo Urbano y Vivienda, GEM.

- Herrera, Fernando (1997), Tesis: Diagnóstico del impacto del turismo residencial en el uso del suelo urbano y su influencia en los asentamientos humanos irregulares, en la ciudad de Cuenavaca. Mor. Facultad de Turismo, UAEM.

- Hiernaux, Daniel (2005), Estudio del turismo de segundas residencias en México. UAM.

- Instituto Nacional de Estadística, Geografía e Informática (2000), Población ocupada por municipio, sexo y ocupación principal, y su distribución según sector y actividad, México.

- Montaner, Jordi, et, al. (1998), Diccionario de turismo, Ed. Síntesis, España.

- OMt (1995), Estudio económico del turismo mundial: el turismo en la crisis económica y el promedio de la economía de los servicios, España.

- Osorio García, Maribel (2004), Turismo de segundas residencias en México estudio de caso: Ixtapan de la Sal, Estado de México.

- Ricaurte Quijano, Carla V. (2001), Turismo, Sustentabilidad y Gestión Local en el Municipio de Ixtapan de la Sal. Toluca: Tesis de Maestría, Facultad de Turismo, UAEM.

\section{Fuentes electrónicas}

- www.granreserva.com.mx 